\title{
UNA EXPLICACIÓN A LOS MECANISMOS COGNITIVOS QUE PROCESAN EL ADUCTO EN RELACIÓN CON EL DESARROLLO DE UNA LENGUA EXTRANJERA
}

\author{
ANa Bocanegra Valle \\ Universidad de Cádiz
}

«El estudio del aprendizaje de lenguas extranjeras que, en un principio, estuvo intimamente ligado a la problemática de la enseñanza ha adquirido una dimensión empírico-teórica que se ha desarrollado en los últimos años y que, sin dejar de tener valor, si bien indirectamente, para la realidad didáctica, ha dado lugar a un campo de investigación que trata de definirse a sí mismo, de forma independiente, como una disciplina más de las ciencias cognitivas» (LICERAs, 1992: (1).

\section{RESUMEN}

Este artículo explica desde el punto de vista de la psicología y de la neurobiología cuáles son los mecanismos cognitivos encargados de procesar el aducto durante el desarrollo de una lengua. La principal intención de tal análisis radica en conocer la razón o razones por las que el aducto al que un individuo está expuesto no siempre resulta satisfactoriamente procesable, así como qué circunstancias mar- 
can el procesamiento natural de información. Las conclusiones se aplicarán al contexto de lenguas extranjeras y al desarrollo de la lengua dentro del aula.

\section{Planteamiento Inicial}

En el número especial que en junio de 1994 la revista Studies in Second Language Acquisition dedicara a los fundamentos cognitivos de la adquisición de segundas lenguas, sus editores llaman la atención sobre la poca investigación interdisciplinar existente entre la ciencia cognitiva y la adquisición de segundas lenguas así como el poco esfuerzo dirigido hacia este campo por parte de los científicos cognitivos '.

Para entender cómo el ser humano desarrolla una lengua además de la materna, el concepto de aducto (input) adquiere una importancia extrema. Por aducto se entienden aquellos datos lingüísticos a los que simplemente está expuesto un individuo, en oposición a aducto procesado (intake, según Corder, 1967; o comprehensible input, según Krashen, 1981) que hace referencia a aquellos datos lingüísticos que ciertamente han sido asimilados por el sujeto e incorporados a su interlengua.

En el caso de una lengua materna, y de acuerdo con los trabajos de Noam Chomsky, se entiende que su desarrollo es común para todos los miembros de la especie al estar dotados de un conocimiento innato universal específicamente lingüístico (Gramática Universal) que se ve activado desde el medio ambiente que le rodea. La mente humana goza de un dispositivo de adquisición del lenguaje (Language Acquisition Device o $L A D$ ) que le capacita para adquirir y producir lenguaje sin la concentración consciente en los aspectos formales. El cerebro humano está determinado biológicamente para procesar la información que recibe, nuestra mente actúa automáticamente ante unas reglas pre-programadas que nos dicta nuestra evolución en el mundo, las procesamos de una forma determinada y aparecen de este modo una serie de resultados

1 «It strikes us, then, how comparatively little interdisciplinary research there is within cognitive science about second language acquisition (SLA). There is, of course, a great deal of research within SLA itself that draws upon research in cognition and extends those ideas in important and interesting ways into both SLA and second language instruction (SLI). Yet SLA has never really taken a particularly prominent place within cognitive science overall. Even a brief glance at recent proceedings of the Cognitive Science Society reveals comparatively little effort directed at SLA. The irony is doubled when one considers that SLA has as a discipline properly taken pride in its multidisciplinary roots. It combines ideas and research strategies from linguistics, sociology, anthropology, education, psychology, and even biology as it examines the myriad difficulties of describing and explaining how individuals fail and succeed in learning additional languages» (TOMLIN $Y$ GERNSBACHER, 1994: 129). 
constantes y universales en el género humano (Guijarro, 1990). Pero ¿qué ocurre cuando esa lengua no es la materna?

En primer lugar habría que distinguir las condiciones que rodean a su desarrollo. En el caso de lenguas segundas el individuo se encuentra en un entorno natural donde está obligado a utilizar la nueva lengua para cualquier fin. En el caso de lenguas extranjeras, el contacto con la lengua meta está limitado a un espacio físico fijo (el aula de clase), a unos interlocutores fijos que implican interacciones fijas (los compañeros y el profesor), y a un tiempo igualmente fijo y concreto (los horarios adjudicados). Todas estas circunstancias restringen el desarrollo natural de la nueva lengua, se tiene conciencia sobre sus aspectos formales, y se limita la facultad innata del individuo de ir construyendo su propio lenguaje de forma creativa. En este sentido, nuestras sospechas en cuanto a las deficiencias que presenta el aula para la posible adquisición de una lengua están relacionadas con el tipo de aducto que recibe el aprendiente, lo que nos lleva a intuir que la diferencia observable entre la exposición dentro y fuera del aula de clase puede tener su origen en que los datos que la comunicación habitual en clase le proporciona al cerebro no concuerdan con aquéllos que la mente necesita para funcionar de forma natural. Atendiendo a este razonamiento, sería la falta de concordancia aducto-exposición natural en relación con el funcionamiento cerebral durante el procesamiento de información la que dificulta el desarrollo de la lengua extranjera.

A fin de optimizar tal desarrollo, parece oportuno, pues, conocer cómo la mente humana procesa el lenguaje de forma natural, así como encontrar una explicación a por qué todos los datos lingüísticos a los que el ser humano está expuesto no tienen igual incidencia en su cerebro. Siguiendo a Wolff (1990), los mecanismos de procesamiento del lenguaje son los mecanismos de adquisición, por tanto, si se pudiera llegar a distinguir las condiciones que hacen al aducto procesable, la actividad dentro del aula podría satisfacer en la medida de lo posible las directrices que impone un desarrollo natural y, así, poner de manifiesto la eficacia del aula como entorno de generación y procesamiento de información.

\section{Propuesta Psicológica al Procesamiento del Aducto}

De Jerry Fodor (1983 y 1990) parte la propuesta de tres procesadores psicológicos por los que pasan los datos que entran en nuestro cerebro:

(1) unos sensores orgánicos que responden al flujo de eventos procedentes del entorno (compiled transducers); 
(2) unos sistemas de entrada, también sistemas modulares o sistemas periféricos, que representan la información sobre el mundo recogida por los sensores de manera tal que el cerebro pueda utilizarla y procesarla (input systems); y, finalmente,

(3) unos procesadores centrales, también sistemas generales o zonas de convergencia, a donde llega cierta información para permanecer allí (central processors).

Aceptando esta taxonomía, toda información que entra en el cerebro humano seguiría entonces un curso bien definido: a través de los sensores orgánicos llega hasta los sistemas de entrada, o continúa hasta los procesadores centrales para ser almacenada y procesada. El primero sería el caso del simple aducto, el segundo el del aducto procesado.

De acuerdo con Fodor, la mente humana está organizada en módulos, y los sistemas de entrada son procesadores cognitivos modulares caracterizados por varias propiedades ${ }^{2}$. De entre éstas resulta de particular interés para el análisis del procesamiento del aducto el hecho de:

(a) que sólo exista un acceso central y limitado a las representaciones mentales que los sistemas de entrada procesan; $y$,

(b) que la encapsulación o encasillamiento de la información sea expuesto desde el punto de vista de la restricción de los datos disponibles para la confirmación de hipótesis.

Los sistemas de entrada procesan representaciones con menos información de la que el organismo es capaz, y, al mismo tiempo, carecen de niveles intermedios que sean accesibles a la conciencia, por tanto, que codifiquen aquella informacion que no buscamos. No obstante, ya que estos niveles atienden a una serie de prioridades antes de llegar a los procesadores centrales, podría darse el caso de que estos últimos tuvieran, de algún modo, acceso a la salida de los niveles intermedios. Esta posibilidad, pues, podría justificar la existencia del aducto procesado frente a la simple exposición: los sistemas de entrada, como mecanismos encapsulados, o penetrables según Pylyshyn (1984), tienen un acceso restringido a su soporte cognitivo, lo que indica que

${ }^{2}$ Constituyen una familia de módulos con funcionamiento obligatorio y competencias específicas que procesan a través de un único acceso central y limitado a las representaciones mentales, son rápidos, están encapsulados a la información, tienen salidas superficiales, gozan de una arquitectura neurológica fija, exhiben modelos de fallos característicos y específicos, y, por último, durante su formación y desarrollo exhiben una velocidad y secuencia características. 
de toda la información que entra gracias a un análisis perceptivo, sólo una porción es, en realidad, tomada en consideración. En el camino hacia los procesadores centrales se van situando unos niveles de entrada que atienden a una serie de prioridades. De entre éstos, únicamente los niveles superiores entran a formar parte de los procesadores centrales y los inferiores son rechazados o, en ciertos casos, retenidos a cambio de un considerable esfuerzo memorístico. De acuerdo con esto, podríamos concluir que existen ciertos datos del aducto que entran directamente en los procesadores centrales (datos adquiridos) y otros que, por alguna razón, se van asentando en los sistemas de entrada ocupando un nivel determinado (datos aprendidos).

Si recordamos a Krashen (1981 y 1982), el aprendizaje (entendido como proceso consciente del desarrollo de una lengua) no puede convertirse en adquisicion (entendida como proceso inconsciente), puesto que aprendizaje y adquisición se deben entender como dos modos distintos e independientes de desarrollar una lengua no-materna ${ }^{3}$. Éste fue uno de los aspectos más criticados de su Teoría de la Monitorización (Monitor Theory) principalmente por presentar el conocimiento implícito (adquisición) y el explícito (aprendizaje) como dos compartimentos estancos, carentes de mecanismo alguno que admitiera la posibilidad de filtrar información obtenida por medio del aprendizaje, es decir, como conocimiento explícito (Liceras, 1996). De acuerdo con la concepción fodoriana de unos sistemas de entrada compuestos de varios niveles, lo aprendido sí podría convertirse en adquirido. Del mismo modo, y sobre la base de tales prioridades, podrían explicarse los conceptos de aducto y aducto procesado: mientras el primero consistiría en aquellos datos que entran y se asientan en los diferentes niveles de los sistemas de entrada, el aducto procesado resultaría de aquellos datos llegados a los procesadores centrales, bien directamente, bien tras un previo paso por los niveles superiores de los sistemas de entrada.

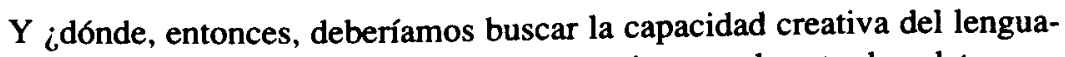
je? Si existen datos que quedan retenidos en los sistemas de entrada y datos que pasan a incorporarse a los procesadores centrales, también se debería prestar atención a todos aquellos datos que nuestro cerebro es capaz de generar sin tener que haber pasado con anterioridad por los sensores orgánicos. En otras palabras, ¿dónde se encuentra el dispositivo innato del lenguaje (LAD) del que

3 Para llegar a esta conclusión, KRASHEN argumentaba, por un lado, que muchos individuos pueden llegar a desarrollar varias lenguas sin conocer sus gramáticas (esto explicaría que puede existir adquisición sin que exista aprendizaje); $y$, por otro, que se puede conocer una regla gramatical y no aplicarla de forma correcta cuando llega el momento (esto explicaría que el aprendizaje no pueda convertirse en adquisición). 
hablaba Noam Chomsky? De acuerdo con Carro et al. (1992) se debería entender el LAD no como un módulo o nivel intermodular sino como el dispositivo que coordina nuestra mente de una forma pre-determinada o determinada genéticamente. Así, la adquisición de una nueva lengua podría entenderse porque «el cerebro de un individuo desarrolla, por exposición a nuevos datos linguíísticos, un nuevo sistema de estructuración (la gramática), gracias a su capacidad de relacionar de forma sistemática, los nuevos estímulos entrantes» (Carro et al., 1992: 41).

No es de extrañar la capacidad de coordinación del LAD puesto que éste fue entendido desde el primer momento como un mecanismo de una capacidad altamente sofisticada que podría desarrollarse, entre otros, gracias a la maduración del sistema nervioso flesto es, gracias a la experiencia del flujo de información entre los procesadores modulares y los centrales, $o$, utilizando las palabras del propio Chomsky (1990), gracias a un crecimiento mental y cognitivo.

Al contrario que los sistemas de entrada, los cognitivos centrales no están encapsulados, y es por esta razón que no son modulares. Su función característica es la de conseguir el proceso inconsciente de fijar creencias (fixation of belief), perceptivas u otras, por medio de inferencias, nunca mediante procesos de código como sería el caso de los sistemas modulares. Con el fin de restringir la computación de las mejores hipótesis acerca de cómo es el mundo, los procesadores centrales acuden, por un lado, a la información proporcionada por los sistemas de entrada, y, por otro, a la que se encuentra residente en la memoria (Guijarro, 1994). En primer lugar, almacenan en la memoria una serie de representaciones que pasan seguidamente a analizar. Tras este análisis, las representaciones se utilizan para realizar operaciones deductivas mediante las cuales se recogen las nuevas representaciones derivadas, producto de los procesos de inferencia, y se eliminan aquellas representaciones anteriores que han pasado a ser consideradas como inservibles. Esto le lleva a Fodor a concluir que los sistemas cognitivos gozan de una estructura neurológica asociada con la percepción y el lenguaje, pero no con el pensamiento. No obstante, lo más importante de toda esta labor de discriminación es, según Guijarro (1994), que para que el ser humano amplíe su visión del mundo y mejore el esquema representacional del mundo que le rodea, ante todo debe aprovechar al máximo todos aquellos datos que el entorno le presenta.

En estos niveles de aprovechamiento es donde podría encontrarse la razón que explique y justifique la labor de discriminación y priorización de los datos durante el procesamiento de la información. Por tanto, ¿cómo se puede explicar la adquisición o incorporación del aducto a los procesadores centrales? ¿Qué hace que el aducto sea adquirido, bien de forma directa, bien previo paso por los niveles superiores de los sistemas de entrada? La Teoría de la Rele- 
vancia o Teoría de la Pertinencia (Relevance Theory) formulada por Sperber y Wilson en 1986 puede proporcionar una respuesta consistente a esta cuestión ${ }^{4}$.

Estos autores siguen a Fodor, en primer lugar, al concebir la mente como una variedad de sistemas especializados, cada uno con su propio modelo de representaciones y computaciones, y, en segundo lugar, al asumir que la facultad del lenguaje constituye un sistema de entrada modular que transporta representaciones sensoras de niveles bajos (memoria a corto plazo, también memoria conceptual según Sneesby, 1994) a niveles más altos (memoria a largo plazo, también memoria enciclopédica según Sneesby, 1994). A los sistemas de entrada, o también procesadores locales, se les atribuye los procesos de código (codificación y decodificación), y a los sistemas centrales, o igualmente procesadores globales, se les atribuye los procesos de inferencia. Para Sperber y Wilson, los sistemas centrales ejecutan una diversa variedad de tareas inferenciales, pero su función principal es, sin duda, procesar las percepciones y/o deducciones en conjunción con los datos ya almacenados —es decir, integrar aquella información procedente de los sistemas de percepción (esto es, los de entrada) con aquella información almacenada en la memoria. En esta labor automática de procesamiento y con el ánimo de generar nuevas representaciones, los sistemas centrales procesan únicamente aquellas representaciones que tengan relación con la información almacenada $\mathrm{y}$, al mismo tiempo, rechazan aquella información que haya sido procesada con anterioridad o que no refuerce representaciones ya almacenadas.

En su modelo de comunicación ostensivo-inferencial ${ }^{5}$, Sperber y Wilson, al igual que Fodor, conciben la inferencia como una forma de fijación de creencia. Si los mecanismos centrales son los que directamente procesan la información, se consigue un esfuerzo de procesamiento mínimo a cambio de unos resultados muy positivos, y esto hace que a los sistemas centrales se les consideran los responsables de un procesamiento de información eficaz que se consigue al maximizar la Relevancia de la información procesada ${ }^{6}$.

4 «The criterion proposed in Relevance is based on a fundamental assumption about human cognition. The assumption that human cognition is relevance-oriented: we pay attention to information that seems relevant to us. Now every utterance starts out as a request for the hearer's attention. As a result, it creates an expectation of relevance. It is around this expectation of relevance that the criterion for evaluating possible interpretations of an utterance is built» (WILSON, 1993: 346).

3 «Ostensive-inferential communication consists in making manifest to an audience one's intention to make manifest a basic layer of information» (SPERBER Y WILSON, 1986: 54).

"An utterance, on a given interpretation, is optimally relevant if and only if: (a) it achieves enough contextual effects to be worth the hearer's attention; (b) it puts the hearer to no gratuitous processing effort in achieving those effects» (WILsON, 1993: 350). De donde efectos contextuales se entienden como el resultado de la interacción de la información reciente con el conjunto de suposiciones que tiene el oyente acerca del mundo. 
Si los procesadores centrales son los encargados de integrar la información procedente de los sistemas de entrada con aquella información que ya está presente en cada representación individual del mundo, y, por tanto, existe en la memoria, se podría decir que lo que tales sistemas hacen es mezclar una información nueva (la de los sistemas de entrada) con una información vieja (la de la memoria) para obtener nueva información. Este efecto marcaría el hecho de Relevancia que aumenta conforme más conexiones de nueva-vieja información se produzcan. Por otra parte, interconexiones aisladas supondrían un gran esfuerzo de procesamiento a cambio de escasos beneficios. La Teoría de la Relevancia adelanta, pues, un modelo de procesamiento del aducto que está dirigido por un así denominado Principio de Relevancia ${ }^{7}$ que gobierna la cognición humana y que relaciona la actividad mental del individuo con el entorno. ${ }^{8}$

El procesamiento de datos aspira en todo momento a una Relevancia óptima, y únicamente se llegan a procesar aquellas hipotesis que parezcan relevantes o pertinentes en un momento dado. Dos cuestiones merecen especial atención:

(1) que los efectos contextuales aumentan y el esfuerzo de procesamiento disminuye en relación con la inmediatez del entorno físico; $y$,

(2) que existe una conexión directa entre la cantidad de efectos contextuales y su repercusión en la memoria/imaginación.

De donde: (1) la inmediatez del entorno físico, y (2) el número de efectos contextuales conseguidos, condicionan la posibilidad de fallo al determinar la selección de una hipotesis compatible, y, por tanto, la posibilidad de que el aducto pase a los procesadores centrales en una primera instancia, o se asiente en alguno de niveles de los sistemas modulares de entrada. La ampliación de suposiciones contextuales iniciará un empuje descendente que alejará a los datos de los procesadores centrales y los situará, dependiendo del número de ampliaciones ocurridas, en alguno de los niveles que componen la franja de los sistemas modulares fla mayor número de ampliaciones, más alejados de los mecanismos centrales y con menos posibilidades de ser adquiridos (Figura 1).

7 El Principio de Relevancia lo formulan SPERBER Y WILSON (1986: 158) del siguiente modo: «Every act of ostensive communication communicates the presumption of its own optimal relevance». $O$ también: «The principle of relevance is the principle that every utterance (or other act of overt communication) creates an expectation of relevance" (WILSON, 1993: 349).

${ }^{8}$ Para entender mejor este modelo y profundizar en ciertos conceptos, consultar Bocanegra Y HAIDL (1997). 


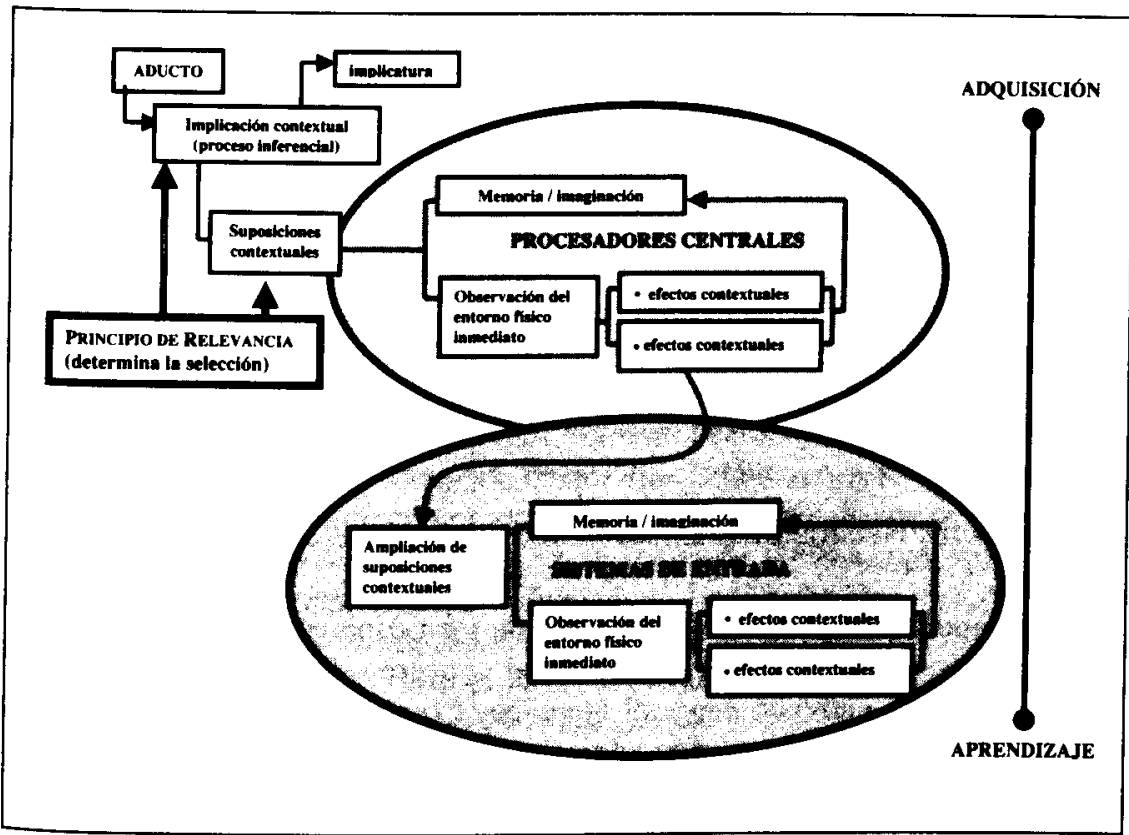

Aceptando, pues, que los mecanismos centrales procesan el aducto directamente y son responsables de los procesos de fijación de creencia, el plan de actuación dentro del aula debería estar dirigido a conseguir procesos de inferencia que posibilitaran el procesamiento del aducto directamente con arreglo a los sistemas centrales o, en todo caso, a los niveles superiores de los sistemas de entrada. Para optimizar el procesamiento del aducto dentro del aula y, consecuentemente, garantizar la adquisición de la lengua meta, toda actividad debe aspirar a conseguir el mayor número de efectos contextuales a cambio de un esfuerzo mental de procesamiento mínimo. El hablante, por su parte, debe crear expectativas de Relevancia (esto es, ostensión), debe provocar inferencias que desarrollen la comunicación, manifestar su intención de transmitir un mensaje para que el oyente deduzca esas inferencias, asuma el deseo del hablante de transmitir, e interprete dicho mensaje. En pocas palabras, el oyente debe encontrarse cognitivamente predispuesto para reconocer la intención del hablante de transmitir Relevancia y procesar de forma eficaz toda la información que recibe. El Principio de Relevancia se hace de este modo manifiesto en ambos extremos del proceso, el hablante manifiesta una Relevancia que el oyente retoma para interpretarla y construir hipotesis compatibles con la mayor cantidad de datos que alberguen los procesadores centrales. 
Un aducto procesado, o aducto que llega a los mecanismos centrales de procesamiento para permanecer allí, es producto del éxito de la comunicación ostensivo-inferencial en la que tenga lugar. Cuanto mayor número de veces haya que ampliar los efectos contextuales que rodean a un enunciado inicial, más se irán alejando los datos de los procesadores centrales para asentarse en los diferentes niveles intermedios que componen los sistemas de entrada. Dependiendo de estas necesidades de ampliación, los datos quedarán en niveles superiores o inferiores, lo que traería consigo diferentes grados de procesamiento del aducto y, consecuentemente, de incidencia en la adquisición de la lengua (Figura 1).

\section{Propuesta Neurobiológica al Procesamiento del Aducto}

Las aportaciones que la neurobiología, y en concreto la neuroanatomía y neurofisiología ${ }^{9}$, pueden llegar a hacer al concepto de aducto y su procesamiento también merecen especial atención. Como bien justifican Eubank y Gregg (1995), si los procesos de adquisición de una lengua pretenden explicar ciertos estados y cambios del conocimiento y, ya que el conocimiento está establecido en el cerebro, las teorías de adquisición son también, por definición, teorías de los estados del cerebro y de los cambios en dichos estados.

La preocupación por estudiar cuál es el papel del sistema nervioso, concretamente del neocórtex ${ }^{10}$, en el desarrollo del lenguaje tanto verbal como noverbal ha llevado a definir el aprendizaje como un proceso íntimamente ligado

9 «eurobiology refers to the study of the nervous system in all species, including how the nervous system interacts with the environment. Neurobiological findings, especially those related to plasticity, memory, and learning, apply at a basic level to the language acquisition process. Neurobiology may be divided into several interrelated subdisciplines, the principal areas being neuroanatomy, neurochemistry, and neurophysiology. Neuroanatomy is concerned with structural architecture, which is necessary for the integration of other subdisciplines (for example, neuropharmacology), and provides a powerful starting point for functional inferences (...) Neurochemistry examines chemical systems and processes (for example, protein synthesis) and helps to explain how nerve cells function and communicate with each other (for example, via neurotransmitters). Neurophysiology focuses on the interaction (i.e. function) of the various components of the nervous system and describes how nerve cells respond to input and conduct signalsm (JACOBS Y SCHUMANN, 1992: 283-4).

${ }^{10}$ El neocớtex es la materia gris que envuelve el sistema límbico. Está dividido en dos hemisferios, izquierdo y derecho, y constituye la conocida corteza cerebral que ocupa un $85 \%$ de la masa cerebral. Podemos definir sistema límbico como «la estructura nerviosa más importante implicada en la conducta emocional, de los impulsos y tendencias instintivas, afectos y estados de ánimo» (CARRO Et Al., 1992: 38). 
al funcionamiento de las neuronas: la liberación de los transmisores neuronales o neurotransmisores, mediante la modulación de los canales de calcio de terminales presinápticos, constituye la base fisiológica y molecular del cerebro humano y, por tanto, también del aprendizaje (Eubank y Gregg, 1995). Además del sistema cerebral, el sistema límbico participa en las diferentes fases de la memorización, tanto filtrando información, como discriminando estímulos mediante el envío de datos a toda la corteza cerebral. Las conexiones neuronales que forman el sistema límbico son las responsables de la conducta emocional del individuo, y, lo que es más importante, de la adquisición del lenguaje (Pulvermüller y Schumann, 1994).

El hecho de que nuestra estructura cerebral pueda afectar a la cognición se debe al funcionamiento de una parte del sistema límbico, conocida como amígdala, que evalúa el significado emocional y motivacional de los estímulos para influir tanto en la atención como en la memoria (Mishkin y Appenzeller, 1987). Al mismo tiempo, tal sistema de evaluación de estímulos modula la cognición y condiciona su desarrollo (Schumann, 1994) lo que hace imprescindible una aproximación a la neurobiología si realmente se tiene interés en estudiar los procesos cognitivos que sustentan la adquisición de una lengua.

Los sistemas de información del sistema nervioso están compuestos de tres vías o zonas que, a nuestro entender, encuentran fiel reflejo en la división fodoriana del cerebro. Así, la neuroanatomía distingue entre:

(a) receptores, que responden a los estímulos externos y, como tales, corresponderían a los sensores orgánicos;

(b) vias aferentes, que se ocupan de llevar la información al cerebro del mismo modo que los sistemas de entrada le proporcionan al cerebro información sobre el mundo para que éste pueda utilizarla;

(c) áreas sensoriales, donde se integra la información procedente de las vías aferentes y que, al igual que los procesadores centrales, es la zona a la que llega cierta información para permanecer allí.

Asimismo, los ya conocidos diferentes niveles intermedios situados desde los módulos de entrada hasta los sistemas centrales de procesamiento podrían tener sus correspondientes localizaciones neuroanatómicas. De acuerdo con Carro et al. (1992), gozamos de: una memoria inmediata, que mediante un esfuerzo mantiene activos los circuitos neuronales; una memoria reciente, que igualmente activa los circuitos neuronales durante algunos minutos más; y, finalmente, una memoria remota, que tras la consolidacion de las sinapsis hasta el momento implicadas, se almacena de forma temporal en el neocórtex del hemisferio derecho. De aquí, podríamos concluir que memoria inmediata y re- 
ciente (short-term memory) corresponderían a los módulos de entrada anteriores, y la memoria remota (long-term memory) a los procesadores centrales.

La ya conocida división del neocórtex en dos hemisferios, derecho e izquierdo, dio origen en su momento a todo tipo de discusiones referentes a las competencias específicas de cada uno de ellos. El hemisferio izquierdo era concebido como la mitad logica y consciente donde se alojaba la comunicación verbal, y el hemisferio derecho la mitad emocional e inconsciente correspondiente a la comunicación no-verbal; de este modo, la comunicación verbal era entendida como lógica mientras que la no-verbal un simple producto emocional del hemisferio derecho. Con el paso de los años se ha llegado a la conclusión de que ambos hemisferios funcionan de forma complementaria en la cognición humana (el izquierdo analíticamente y el derecho sintéticamente) pero complementándose, no excluyéndose, el uno al otro ".

En este punto habría que destacar las aportaciones de MARCEL DANESI (1986, 1987a, 1987b, 1988a, 1988b) al estudio de la neurolingüística. Preocupado por conocer cómo selecciona y maneja el cerebro humano el aducto verbal en el desarrollo de una lengua y las implicaciones de tal funcionamiento para con el trabajo dentro del aula, Danesi presenta un enfoque didáctico o marco de trabajo que él mismo denomina enseñanza de lenguas compatible con el funcionamiento cerebral ('brain-compatible' language teaching) o también bimodalidad (bimodality) ${ }^{12}$. Según este enfoque, la única fórmula de enseñanza compatible con el funcionamiento natural del cerebro es aquélla que atiende a la direccionalidad hemisferio derecho-izquierdo pues resulta ser este último el que finalmente controla y evalúa el aducto. Esto implica una necesidad de estudiar y conocer las funciones intuitivas del hemisferio o módulo derecho (Right $M o d e$ o $R$-Mode) durante una primera etapa de generación de ideas para más tarde pasar, en una etapa posterior de formalización, a las funciones secuenciales y organizativas del hemisferio o módulo izquierdo (Left Mode o L-Mode).

"Las dos mitades del cerebro trabajan de forma complementaria en el sentido de que una toma control sobre la otra en el momento en que se siente con capacidad de resolver un problema concreto. Las cargas de procesamiento analítico y sintético se distribuyen entre ambos hemisferios para evitar una posible fatiga cerebral, o sobrecarga cognitiva según RIVERs (1994), de manera similar a lo que ocurre cuando nos pasamos una maleta pesada de una mano a otra para evitar el cansancio de un miembro concreto de aquí, la denominación de 'suitcase' theory a la participación conjunta de hemisferios izquierdo y derecho en toda actividad neurológica.

12 «Bimodality is proposed as an educational construct which attempts to translate the neurophysiological concept of hemispheric complementarity or cooperative interaction into practical classroom implications. It can be defined as the notion that posits that for language learning to become a global communicative process instructional practices must exploit the traits of both hemispheres in a complementary fashion (...) It is a simple attempt to translate the hemispheric interaction model into a set of classroom working principlesw (DANESI, 1988b: 460). 
Las palabras de DANESI son tajantes a la hora de garantizar la efectividad en la enseñanza/desarrollo de una lengua extranjera: la metodología adecuada debe atender a los dictados del cerebro humano y, así, trabajar de acuerdo con el ritmo y la direccionalidad que éste impone (Figura 2). El hemisferio derecho es el más sensible a los nuevos estímulos y, dada su naturaleza, ofrece mayores posibilidades de conexión entre las regiones del cerebro, al tiempo que descifra la información sin atender a códigos pre-existentes o programas disponibles. Una vez que el hemisferio derecho ha procesado la información nueva, el hemisferio izquierdo toma el relevo para terminar de procesar tales datos de acuerdo con el sistema identificado (Goldberg y Costa, 1981).

Carro et al. (1992) llaman la atención sobre la costumbre generalizada en el ámbito docente de fomentar en el aula los procesos típicos del hemisferio izquierdo (los conscientes) y no esforzarse por movilizar los inconscientes y emocionales, que resultan igualmente importantes, en particular, para la adquisición. De acuerdo entonces con todo esto, la actividad a desarrollar dentro del aula debe comenzar estimulando el Módulo-R (hemisferio derecho e inconsciente) que presupone adquisición, para pasar más tarde a estimular el Módulo-L (hemisferio izquierdo y consciente), que presupone aprendizaje, de forma que ambos trabajen cooperativa y complementariamente y los procesos neurobiológicos que gobieman la mente humana sean compatibles con la actividad de trabajo dentro del aula.

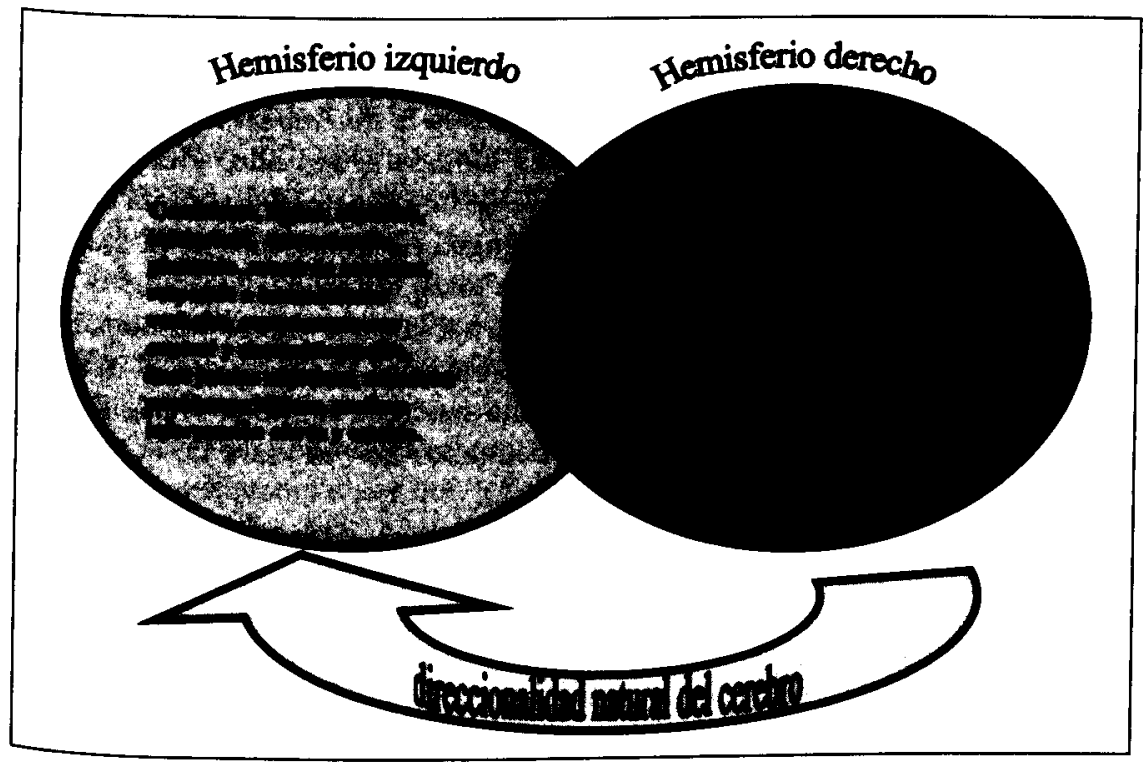


Todas las actividades que se pongan en marcha no deben ser arbitrarias y dedicarse a educar caprichosamente un único hemisferio, ya sea derecho o izquierdo; por el contrario, deben estar motivadas por la bimodalidad. En la unimodalidad es donde radica la explicación neurológica que justifica el fracaso, en muchos casos, de una metodología de enseñanza de una lengua (Levy, 1985). Todos conocemos casos en los que se sabe a la perfección una regla gramatical pero no se aplica con propiedad en los casos correspondientes; esto ocurriría porque tal conocimiento consciente se aloja en otras bases (Timm, 1991), instancias (Carro et al., 1992) o módulos (Fodor, 1981) mentales que no son responsables directos del habla.

Una metodología donde las coordenadas neurobiológicas definan una marco de acción ayudará a trazar la dirección adecuada de trabajo a seguir. Johnson (1985) y Arndt et al. (1986) hacen una importante aportación a la direccionalidad y competencias de trabajo de ambos hemisferios: el izquierdo está especializado en un procesamiento de información secuencial, además de analítico, mientras que el derecho lo está en un procesamiento simultáneo, además de sintético. Partiendo de este hecho, Mohan (1986) propone el movimiento de aprendizaje experimental a aprendizaje teórico, de contenido práctico a contenido teórico, de habla práctica a habla teórica flen definitiva, de uso de la lengua a conocimiento metalingüístico.

Puesto que el hemisferio izquierdo, según Danesi (1988b), interpreta el aducto linguístico (texto) y el hemisferio derecho contribuye de forma crucial a la comprensión de las circunstancias que lo rodean (contexto), podemos concluir que la mejor forma de activar el cerebro de manera que funcione como un dispositivo eficaz de procesamiento de aducto, es la de contextualizar los datos lingüísticos para explotar su naturaleza bimodal. Para ello, y como bien apunta Haidl (1993), resulta necesario gozar de un modelo que permita definir o describir las características del uso de la lengua en situaciones de la vida real o, más específicamente, por qué el uso que los aprendientes hacen de la lengua dentro del aula, aún cuando son instados a hablar, «no parece suficiente para producir una automatización de los conocimientos» (p. 376). Con el modelo adecuado se podrían contrarrestar de alguna manera, continúa Haidl (1993: 377), «las desventajas que distinguen la comunicación en clase de la natural, fuera de ella».

Si el hemisferio derecho es el encargado de procesar los nuevos datos que llegan al cerebro al mismo tiempo que maneja mejor el factor contexto, a fin de optimizar el desarrollo de la lengua dentro del aula se deberían poner en marcha las técnicas que conllevan el funcionamiento activo del módulo-R, uniendo las variables de novedad y contexto como punto de partida de las diferentes actividades a realizar. Después de que los aprendientes hayan recogi- 
do todos aquellos datos nuevos de forma inconsciente e intuitiva, se podrá decir que sus sistemas mentales estarán preparados para asignar a tales datos esquemas cognitivos apropiados (DANESI, 1986) o, como afirman Sperber y Wilson (1986), se encontrarán cognitivamente predispuestos. Actividades como aquéllas que conlleven algún tipo de toma de decisiones (problem-solving activities) ${ }^{13}$ resultarán eficaces para activar la función cerebral y fomentar la participación de ambos hemisferios (Genesee, 1988).

Con este esfuerzo común es de esperar que se evite toda sobrecarga cognitiva posible que explicaría por qué muchos aprendientes no son capaces de procesar en el momento adecuado toda aquella información que se encuentra en su memoria. Con un planteamiento en estos términos, para que pueda haber adquisición dentro del aula se debería activar el funcionamiento natural del neocórtex haciendo, ante todo, que el empleo de la lengua meta sea lo más parecido posible al que se hace de él en la vida real: se deberían crear auténticas (no simuladas) necesidades de comunicación y, principalmente, los aprendientes se deberían comprometer o involucrar en el uso que hacen de la lengua durante la negociación del aducto.

\section{PARA CONCLUIR}

La gran diferencia que condiciona el éxito del desarrollo de una lengua segunda frente a una lengua extranjera puede tener su origen en que en el primer caso, al contrario que en el segundo, el aducto se procesa de forma natural en todo momento. Gracias a la psicología cognitiva por un lado y a la neurobiología por otro, parece posible desentrañar las claves que hacen procesables los datos que componen el aducto.

La organización de la mente en instancias o módulos intenta dar una explicación al camino que siguen los datos para llegar a los procesadores centrales. Con arreglo a tal organización, Sperber y Wilson, con su Teoría de la Relevancia y la comunicación ostensivo-inferencial, solucionan el problema de la eficacia en el procesamiento de la información, además de proporcionar una respuesta consistente a por qué los datos a los que un individuo está expuesto tienen desigual incidencia en su cerebro y de qué depende que un aducto sea procesado con eficacia.

13 SPERBER Y WILSON (1986) también entienden que la solución de problemas de cualquier tipo resulta ser un buen modo de procesar información pues su eficacia radica, básicamente, en llegar a un fin concreto con un esfuerzo de procesamiento mínimo. 
Por su parte, la neurobiología nos acerca desde los parámetros de la neurofisiología y la neuroanatomía al funcionamiento de los dos hemisferios que componen el cerebro y su relación con el procesamiento de la información. De acuerdo con tal división, los trabajos de Marcel Danesi abren una nueva luz a la enseñanza/desarrollo de lenguas extranjeras, al afirmar que la efectividad de adquisición de una lengua dentro del aula es proporcional al grado de utilización de una metodología que tenga en cuenta la naturaleza bimodal que gobierna todo aprendizaje humano.

\section{REFERENCIAS}

ARNDT, H.; JANNEY, R.W.; SCHAFFRANEK, G. (1986): «A neurological view of prosody and its importance in human communication». Die Neueren Sprachen, 85, 581-610.

BoCANEGRA, A.; HAIDL, A. (1997): "A Relevance-driven model for input processing». En: DIAZ, L.; PÉREZ, C. (Eds.). Views on the Acquisition and Use of a Second Language. EUROSLA ' 7 Proceedings. Barcelona, Universitat Pompeu Fabra, 395-402.

CARRO, M.; FERnÁNDEZ, J.; GOMEZ, A.; HAIDL, A. (1992): «Bases neurofisiológicas y neuroanatómicas del habla. Su importancia para la adquisición de lenguas extranjeras». Tavira, 9, 27-46.

CHOMSKY, N. (1990): «On the nature, use and acquisition of language». En: LYCAN, w.G. (Ed.). Mind and Cognition: a Reader. Oxford, Basil Blackwell, 627-46.

CORDER, S.P. (1967): «The significance of learner's errors». IRAL, 5, 161-70.

DANESI, M. (1986): «Research on the brain's hemispheric functions: implications for second language pedagogy». Lenguas Modernas, 13, 99-113.

DANESI, M. (1987a): «Applied neurolinguistics: what the language teacher should know about current brain research». Teacher Education, 31, 32-41.

DANESI, M. (1987b): «Practical applications of current brain research to the teaching of Italian». Italica, 64, 377-92.

DANESI, M. (1988a): "Neurological bimodality and theories of language teaching». Studies in Second Language Acquisition, 10, 13-31.

DANESI, M. (1988b): «From context to text: synchronizing language teaching to the neurology of language learning». Die Neueren Sprachen, 87, 454-70.

EubANK, L.; GreGG, K.R. (1995): “'Et in Amygdala ego?’, UG, (S)LA, and Neurobiology». Studies in Second Language Acquisition, 17, 35-57.

FoDOR, J.A. (1981): Representations. Cambridge, Mass., MIT Press.

FODOR, J.A. (1983): The Modularity of Mind. Cambridge, Mass., MIT Press.

FODOR, J.A. (1990): «Why should the mind be modular?» En: GEORGE, A. (Ed.). Reflections on Chomsky. Oxford, Basil Blackwell, 1-22. 
GENESEE, F. (1988): «Neuropsychology and second language acquisition». En: BEEBE, L.M. (Ed.). Issues in Second Language Acquisition: Multiple Perspectives. Rowley, Mass., Newbury House, 81-112.

GOLDBERG,E.; COSTA, L.D. (1981): «Hemispheric differences in acquisition and use of descriptive systems». Brain and Language, 14, 144-73.

GuiJarRo, J.L. (1990): «Perspectiva de una pasión ornitológica desde un ala delta, o: algunos problemas cognoscitivos ante el punto de vista». Estudios de Lengua y Literatura Francesas, 4, 59-72.

GujaRRo, J.L. (1994): «Comunicación literaria». En: Ruz Castellanos, A. (Ed.). Actas del Primer Encuentro Interdisciplinar sobre Retbrica, Texto y Comunicación. Cádiz, Servicio de Publicaciones de la Universidad de Cádiz, 110-20.

HaidL, A.w. (1993): «La 'Teoría de la Relevancia' y los procesos de adquisición en la enseñanza de idiomas extranjeros». Pragmalingüística, 1, 367-98.

JACOBS, B.; SCHUMANN, J.H. (1992): «Language acquisition and the neurosciences: toward a more integrative perspective». Applied Linguistics, 13, 282-301.

JOHNSON, V.R. (1985): «Myelin and maturation: a fresh look at Piaget». En: WRIGHT, O.M. (Ed.). Readings in Adolescent Development. Toronto, Board of Education, 57-62.

KRASHEN, S.D. (1981): Second Language Acquisition and Second Language Learning. Oxford, Pergamon Press.

KRASHEN, S.D. (1982): Principles and Practice in Second Language Acquisition. Oxford, Pergamon Press.

LEVY, J. (1985): «Right brain, left brain: fact and fiction». Psychology Today, 19, 38-44.

LICERAS, J.M. (1992): «Hacia un modelo de análisis de la interlengua. Introducción». En: LICERAS, J.M. (Ed.). La Adquisición de las Lenguas Extranjeras. Madrid, Visor, 11-27.

LiCERAS, J.M. (1996): La Adquisición de las Lenguas Segundas y la Gramática Universal. Madrid, Editorial Síntesis.

MishKIN, M.; APPENZELlER, T. (1987): «The anatomy of memory». Scientific American, 256, 80-9.

Mohan, B.м. (1986): Language and Content. Reading, Mass., Addison-Wesley.

Pullvermüller, F.; SChumanN, J.H. (1994): «Neurobiological mechanisms of language acquisition». Language Learning, 44, 681-734.

Pylyshyn, Z. (1984): Computation and Cognition. Cambridge, Mass., MIT Press.

RIVERS, W.M. (1994): «Empowering the learner for effective communication». Conferencia de inauguración del $\mathrm{V}$ Congreso Luso-hispano de Lenguas Aplicadas a las Ciencias, Septiembre 29-30 y Octubre 1,Valencia.

SChumanN, J.H. (1994): «Where is cognition? emotion and cognition in second language acquisition». Studies in Second Language Acquisition, 16, 231-42.

SNEESBY, P. (1994): La Producción Escrita del Inglés por Medio de los Programas de Ordenador: Principios Básicos para Asegurar la Enseñanza y el Aprendizaje. Tesis Doctoral. Universidad de Cádiz. 
SPERBER, D.; WILSON, D. (1986): Relevance: Communication and Cognition. Oxford, Basil Blackwell.

TIMM, J.P. (1991): «Englischunterricht zwischen Handlungsorientierung und didaktischer (ein Nachtrag)». Die Neueren Sprachen, 90, 559-63.

TOMLIN, R.S.; GERNSBACHER, M.A. (1994): «Introduction to cognitive foundations of second language acquisition». Studies in Second Language Acquisition, 16, 129. 32.

WILSON, D. (1993): «Relevance and understanding». Pragmalingüística, 1, 335-65.

WOLFF, D. (1990): «Zur Bedeutung des prozeduralen Wissens bei Verstehens -und Lemprozessen im schulischen Fremdsprachenunterricht». Die Neueren Sprachen, 89, 610-25. 